\title{
A Logistics Monitoring Technology Based on Wireless Sensors
}

\author{
https://doi.org/10.3991/ijoe.v13i07.7375 \\ Hongxia Sun \\ Zhengzhou Shengda University of Economics, Business \& Management, Zhengzhou, China \\ sunhongxia421@163.com
}

\begin{abstract}
A logistics monitoring technology is proposed based on the analysis for the traditional logistics enterprises. By tracking the existing monitoring system and data transmission technology, a new technology WSN-based logistics vehicle monitoring system is adopted. The practical significance to monitoring and managing the logistics processes effectively is to diminish transportation costs, improve logistics efficiency and avoid needless economic loss. The results show that the WSN not only realizes the positioning of the vehicle, but also can effectively manage the goods, improve the efficiency in the transportation management of the goods. In addition, this experiment system also adds a temperature sensitive alarm circuit to improve the safety performance of the terminal. Based on the result, it is concluded that this technology can adjust the transmission power and reduce energy consumption to extend the network life cycle as long as possible.
\end{abstract}

Keywords-WSN, logistics monitoring, GPS

\section{$1 \quad$ Introduction}

The logistics vehicle monitoring system is an information system which comprehends information collection, wireless communication and data processing and some other techniques to monitoring, controlling and dispatching logistics vehicles and merchandises. The system is generally running in the transportation process, and the monitoring object is vehicles or merchandises. The logistics vehicle monitoring system is ordinarily composed of vehicle (cargo) monitoring system, data transmission system and monitoring and management system.

According to the actual needs of logistics enterprises, the characteristic for logistics vehicle monitoring system is stability, timeliness, security and integrality.

\section{WSN technology}

To initiate the application of WSN and RFID and some other IoT (Internet of Things) technology in logistics and transportation monitoring is a tendency at present [1]. 
WSN-based logistics vehicle monitoring system can achieve the real-time state of logistics vehicles and merchandises by installing a large number of wireless sensor nodes in the vehicles, which realize the real-time intelligent monitoring and deliver the data to the monitoring management center through the communication system and provide effective protection for logistics transport vehicles. Typical Internet of Things technology is RFID, Zigbee, WSN, etc., in which the WSN application is the most common [2].

WSN is a self-organized network composed of multiple sensor nodes with wireless communication capability. It is a network information system which integrates distributed information collection, information transmission, and information processing. It can realize the quantization, fusion, and transmission of data collection process. The characteristics of WSN are that it is limited capacity, in large scale, distributed, selforganization and low energy consumption. Generally speaking, WSN communication protocol consists of the physical stage, network stage, data link stage, transmission stage, and application stage.

As shown in Figure 1, the physical stage is the substructure for the entire open system and is responsible for signal modulation and reception and transmission of the wireless signal. The network stage is responsible for route generation and selection. The Data link stage is ordinarily responsible for the frame detection, data framing, media access and error control. The research on the stage focuses in the MAC protocol. The transport stage is an important joint to ensure the quality of communication services. The mainly responsibility is to carry data flow control, providing the establishment, maintenance, cancellation of connection for WSN nodes and data error recovery.

The application stage is the application software primarily based on monitoring tasks. The fundamental function of the application stage is to provide a variety of user-oriented application services, such as the monitoring control system, environmental monitoring system, and wild animals tracking system [3].

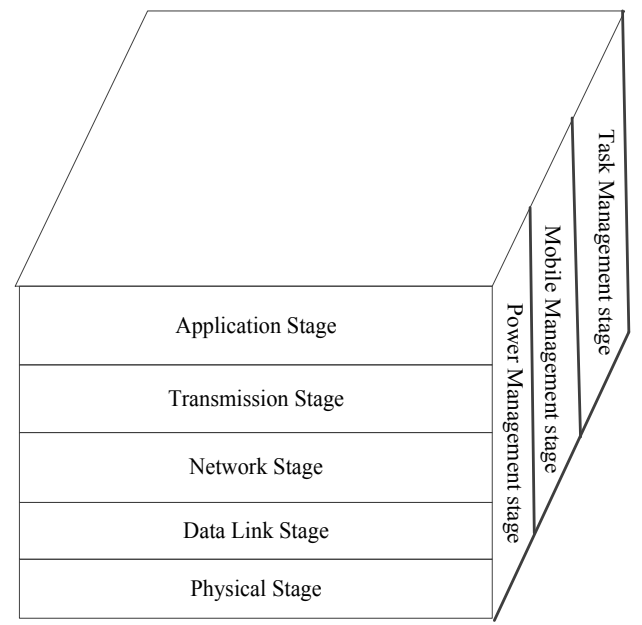

Fig. 1. Protocol Stack Model for Wireless Sensor Network 


\section{$3 \quad$ Materials and Methods}

\subsection{General description of the system}

The primary goal to implement the system is to realize the whole process monitoring and real-time report to enhance the coverage of information collection and the stability of information transmission, to ensure the safety for the vehicles and merchandise and to improve the efficiency and level of logistics management, with the advantage of WSN technology which can carry on on-the-spot supervision of vehicles and merchandise. The overall process of the system application is as follows: In the transportation process, according to the requirements of customer or selfmanagement, the logistics transportation enterprise collects the real-time status of the vehicle cargo (such as temperature, humidity and inclination and etc. parameters) by WSN technology, transmits the data to the remote monitoring and management center. The automatic prompt and advanced warning will be generated if it is necessary, and the monitoring and management center can issue the control instruction to the monitoring system in remote condition at any time.

Besides the features of the general logistics vehicle monitoring system such as stability, timeliness, security and integrality, a WSN-based logistics vehicle monitoring system also has the following characteristics: (1) Automation. The monitoring system can prompt and alarm automatically when there is abnormal circumstance during the monitoring system working. (2) Energy constraints. Since the WSN nodes are generally small with limited energy storage, it is necessary to adjust the transmission power, reduce energy consumption to extend the network life cycle as long as possible.

\subsection{System architecture}

A whole framework of the WSN-based logistic vehicle monitoring system includes three functional subsystems, which are vehicle (cargo) monitoring system, data transmission system, and monitoring and management system respectively

Vehicle (cargo) monitoring system. Vehicle (cargo) monitoring system is primarily responsible for the perception, collection and temporary saving of environmental information. For the perspective of composition, during the logistic vehicle monitoring system, the vehicle (cargo) monitoring system is consist of wireless sensor monitoring network, network coordinator, GPS nodes and other equipment.

Wireless sensor monitoring network. Wireless sensor monitoring network is a selforganized network with a large number of wireless sensor nodes in the compartment, which realize the coverage of the vehicle interior. Wireless sensor monitoring network is the core of the entire vehicle monitoring system. Wireless sensor detection network is consists of multiple nodes and sink nodes and gateways, and which is responsible for the acquisition of the status of the merchandises, and the initial detection of abnormal behavior. The abnormal results of merchandises detection will transmit to the monitoring center in real time. Wireless detection nodes can be installed in fixed or random spots of the interior of logistics transport vehicle. According to different functions, the wireless detection nodes can be divided into sink nodes 
and normal sensor nodes. According to different types, the wireless detection sensors can be divided into infrared sensors, temperature and humidity sensors, smoke sensors, and pressure sensors.

Each sensor node in the vehicle is usually a micro-embedded system, with relative weak storage capacity, processing capacity and communication capacity, powered by a limited battery. The Sampling is taken in a certain frequency by sensor nodes for the cargo. A built-in processor in sensor collects the data to analysis. From the aspect of network function, each sensor node needs to collect local information, store and integrate the data forwarded by other nodes, and complete some specific tasks cooperating with other nodes.

The sensor nodes hardware system is generally composed of four parts: perceptual module, communication module, data processing module and power supply module. The perceptual module is used to perceive and obtain external information, and convert the collected information into a digital signal and transfer it to data processing module. The data processing module is responsible for processing and storing the raw data obtained by the perceptual module, and coordinating and controlling working modes of other modules. The communication module is used to implement communication with other sensor nodes or sink nodes. There are four states of communication module: send, receive, idle and sleep. The energy consumption of sending state is the largest and is the smallest for the sleeping state. When there is the abnormal status for merchandises, the wireless sensor node will transmit the monitoring data of merchandises to the sink nodes by using the wireless communication module. The power supply module is responsible for providing energy to all the modules in the sensor nodes, usually use battery-powered.

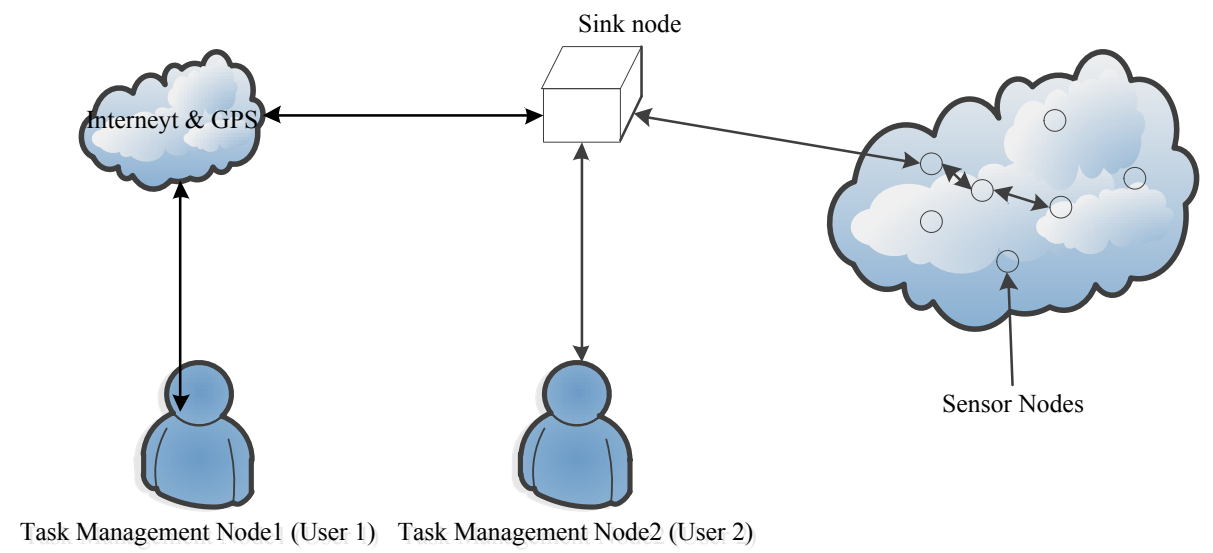

Fig. 2. Structure Diagram of Wireless Sensor Monitoring Network

The common wireless sensor nodes and the sink nodes form the wireless sensor network in the vehicle. A sink node has strong communication capacity, storage capacity and processing capacity, and it can be either a special device with the monitor- 
ing function or an enhanced sensor node. The conversion of sensor network and the Internet or any other external network protocol can be achieved through sink nodes. A sensor node is usually a micro-embedded system that monitors the surrounding environment. The sensor nodes transmit the information to the sink node according to the routing protocol.

Network coordinator. The network coordinator consists of a local module, a power supply module, a processor module and an early warning module. In order to receive the satellite signal normally, the network coordinator antenna needs to be installed leading out of the vehicle, and measured the current location of the vehicle regularly. The variety collected information communicates through the $3 \mathrm{G}$ module and monitoring center of the network coordinator and is sent to the monitoring center. In addition, the network coordinator can also function as a WSN gateway and generally deployed at the top of the compartment of a vehicle.

GPS nodes (or other satellite navigation nodes). The GNSS nodes of the logistics vehicle generally refer to the GPS receiver. The machine is an instrument that receives GPS signals and determines the location of the ground space, with the capacity of receiving, tracking, transforming and measuring GPS signals. This hardware is not the necessary device of the logistics vehicle monitoring system researched in this paper. During navigation and positioning for the logistics vehicles, the built-in antenna will receive the data from the information transmitted by satellite, combining the electronic map in the vehicle navigation to locate the exact spot of the vehicle.

The data transmission system. The data transmission system is carrying on transmitting bidirectional data from WSN and monitoring management, which transmits the information taken from the data acquisition stage to monitoring and management center, and delivers the instructions to the logistic vehicles. Data transmission system hardware includes the base station, relay nodes and other wireless communication devices. Considering the system hardware generally takes uses of the social infrastructure, this article does not make a detailed introduction.

Monitoring and management system. Monitoring and management system is not only a data processing center but also an integrated application management which is in charge of real-time data processing and issue command management. Monitoring and management system is commonly located in corporate headquarter, and its hardware contains the data receiving device ( $3 \mathrm{G}$ communication module), data storage devices and computer terminals or handheld mobile terminals.

Typically, the monitoring center is the control center of the entire logistics vehicle monitoring system. The mass data acquired from the data transmission stage is stored and analyzed in a real-time by the system, and the command management and control is issued during this process. The monitoring software is an important part which supporting the running of monitoring center. As Figure 3, the structure of a general monitoring software function is as follows. 


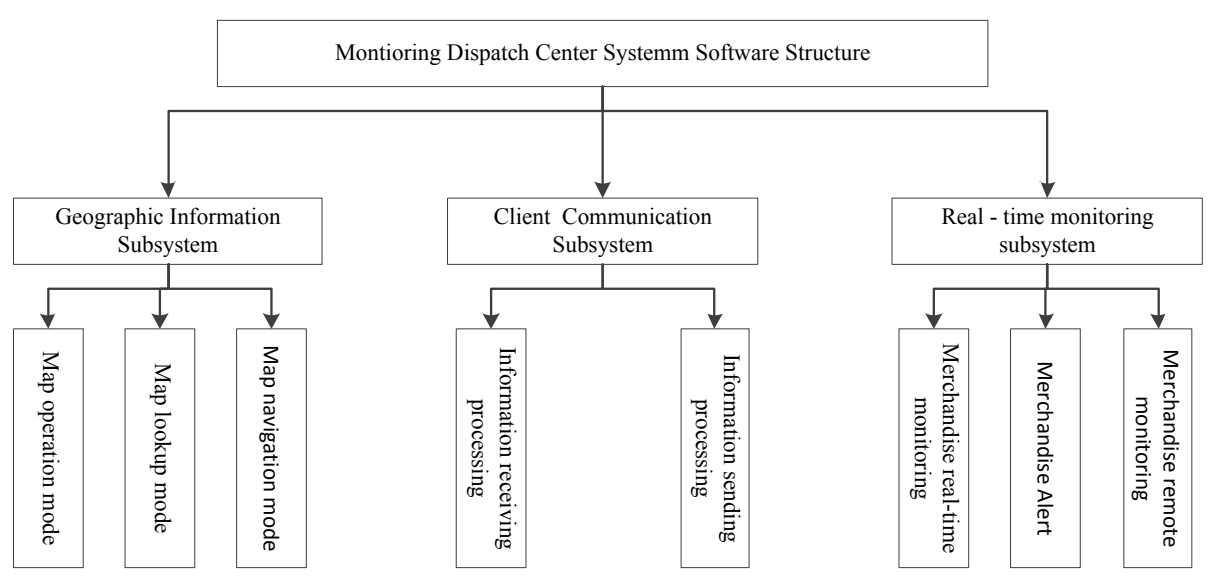

Fig. 3. Structure of Software System for Monitoring Center

\section{$4 \quad$ Logistic Vehicle Monitoring System in Application}

This paper focuses on three aspects of the WSN-based logistic vehicle monitoring system to improve the monitoring: coverage efficiency, mobility management, and QoS routing. The contents of this paper mainly concentrate on the information collection and data transmission of vehicle monitoring system.

1. Coverage efficiency. Mainly refers to study how to minimize the energy consumption and maximize the function efficiency in the condition which ensures the effective coverage with WSN nodes for vehicle and cargo in the logistics compartment. The overlay management is to study the coverage efficiency of wireless sensor nodes inside the logistics vehicle.

2. Mobile management. Mainly refers to study how to realize the stability of communication quality and reduce communication delay with WSN nodes inside the logistics vehicle when the logistics vehicle moves.

3. QoS routing. Mainly refers to study how to improve the transmission efficiency between the sensor nodes by making full use of communication bandwidth inside the logistics compartment.

At present, WSN technology has been put into practice by more and more logistics companies to use improving production efficiency and control of logistics processes. From the operation status of the production enterprises and logistics business, storage environment monitoring, inbound merchandise tracking, dangerous merchandise monitoring, these three aspects are three of the most common subdivision areas of WSN logistics production. WSN with its own unique advantages will have a more wide-spread application with a further study, and the wireless sensor network technology has the main contribution to smart logistics in the collection and receiving process of the information. 
Monitor on the way. The wireless sensor network can be taken into practice in the whole process of tracking and monitoring of transport vehicles and materials. The sensor node can monitor the position and status of each item in transit in real time, and send the flow and status information of the merchandises to the monitoring. The sensor network can be deployed according to the monitoring requirements of different transport vehicles and merchandises, and take sensors which are suitable for their own collection tasks, such as temperature, humidity, acceleration and gas sensors. Logistics companies using the sensor network can get a lower cost to judge and track the goods, equipment, items. On the one hand, the logistics business managers can allocate and manage the logistics processes reasonably with tracking the goods, such as the preparation and transformation. On the other hand, by monitoring the transport conditions of the cargo equipment or merchandises, the logistics company can avoid the probable damage to the goods during the transportation process.

Dangerous merchandises monitoring. Dangerous merchandises monitoring, including on-site monitoring and static monitoring. The dangerous merchandises monitoring system in transit requires controlling the relevant parameters of the merchandises in transit real-time, which ensures the safety of person and equipment and the merchandises arriving in time. It is a more complex monitoring relatively. The monitoring of the merchandises consists of two parts' the vehicle monitoring system and remote management. Vehicle monitoring system is generally controlled by the vehicle control center and a large number of WSN nodes, which built a wireless sensor network.

Cold chain logistics monitoring. In cold chain logistics, the merchandises are usually influenced by external temperature, humidity, and other conditions, which may get a metamorphic situation. In order to maintain the quality and the value of perishable merchandises, it is necessary to store the merchandises at a lower temperature which can satisfy the freshments for their preservation. In this case, the application of WSN technology in the cold chain logistics can monitor the freezing environment of the product temperature and humidity, which can facilitate the timely control of logistics management to ensure product quality for the logistics staff.

\section{$5 \quad$ Results}

To have a further validation of the performance here, we will compare the two different motion models.

In order to verify the positioning accuracy of the beacon under different motion models, here we contrasted the moving beacons to the positioning error of nodes in the network scan (SCAN) AND random waypoint (RWP) under the two motion models and analyzed the factors that influence the position accuracy.

As shown in Figure 4, we compared the positioning accuracy of the nodes under the two motion models at different communication radii. The dynamic beacon speed set as $1 \mathrm{~m} / \mathrm{s}$, communication distance as $10-30 \mathrm{~m}$ and interval distance as $5 \mathrm{~m}$ incremental for the simulation. The results of simulation displayed the positioning error of the nodes under the two kinds of motion models is very close, and the RWY model has a 
slightly higher positioning accuracy. The average positioning error of the nodes in the motion model increases slowly with the increase of the transmission radius, but has a very small influence for the average positioning error. The reason is what we took to locate the nodes is independent to the transmission distance of the nodes.

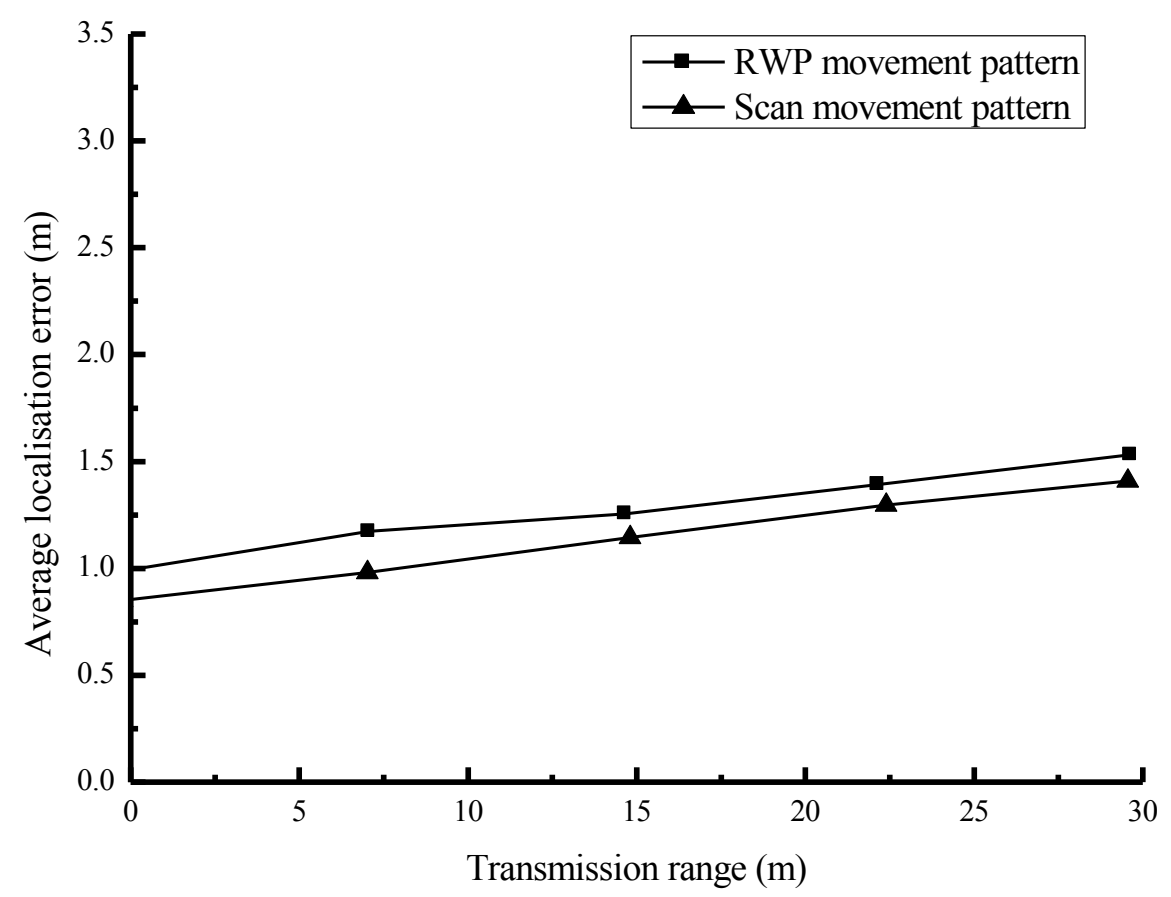

Fig. 4. Average positioning error of SCAN and RWP

Figure 5 shows the impact of different transmission radii for the accuracy of location rates in two kind of motion models. With the simulation results, the node positioning rate of SCAN mobile model has achieved $100 \%$, that is because SCAN uses a full traversal model of the network which ensures all nodes can receive high-quality beacon points. The node positioning rate of RWP increases obviously with a larger transmission radius. The reason is that a random waypoint mobile model cannot guarantee that moving beacons traverse the entire network, but with the transmission radius increases, more nodes will receive the beacons point, and thus make a larger quality of location for nodes.

In order to verify the effect of different beacon intervals for the average positioning error of nodes under the two motion models, we set the broadcast interval at a fixed $1 \mathrm{~s}$, the moving speed varies between $1-5 \mathrm{~m} / \mathrm{s}$, that is, the beacon interval is between $1-5 \mathrm{~m}$ with every 0.5 meters incremental to simulation for multiple times. 


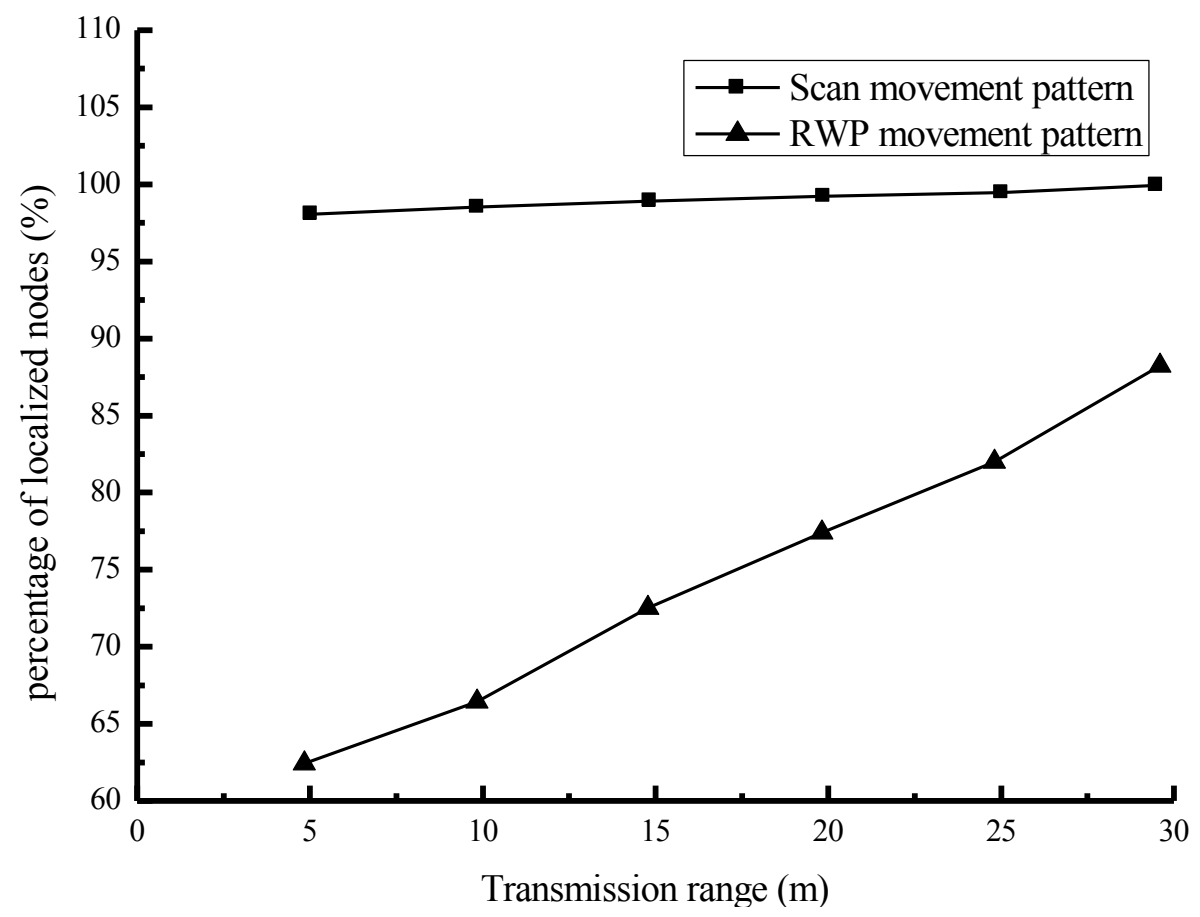

Fig. 5. Percentage of localized nodes of SCAN and RWP

\section{Conclusion}

Logistics monitoring system is a high-tech system which integrates WSN technology, positioning technology and wireless data communication technology. The experiment researches and practices the application technology and wireless access theory of the logistics terminal monitoring experimental system, and draws the following conclusions. First, taking GPRS as the communication link carrier monitoring terminal system, the mobile terminal will be able to enjoy the full access to the Internet function. Second, the results of the moving beacon are simulated and the SCAN and RWP two kinds of motion models are compared. This algorithm reduces quantity of information in transmission and computation information, thus reducing the network energy consumption for WSN technology. Third, short message system overcomes the disadvantages of law transmission speed and improves the reliability of the whole system. Combined with SCAN technology and RWP technology, it has enriched the flexibility and operability of logistics and transportation monitoring. The design scheme based on WSN technology not only realizes the positioning of the delivery tools, but also manages the goods loaded effectively, and achieves the purpose of timely detection of errors and timely processing. 


\section{$7 \quad$ References}

[1] Zhou, Y.H. , Xie Y., and Shao L. (2016). Simulation of the Core Technology of a Greenhouse-Monitoring System Based on a Wireless Sensor Network. International Journal of Online Engineering, 12(5):43-47. https://doi.org/10.3991/ijoe.v12i05.5735

[2] Antolín, Diego, N. Medrano, and Calvo. B. (2016). Reliable Lifespan Evaluation of a Remote Environment Monitoring System Based on Wireless Sensor Networks and Global System for Mobile Communications. Journal of Sensors, 2016:1-12.

[3] Yang, Jiachen, et al. (2015). A Real-Time Monitoring System of Industry Carbon Monoxide Based on Wireless Sensor Networks. Sensors, 15(11):29535-29546. https://doi.org/10.3390/s151129535

[4] González, Iván, et al. (2015). An Ambulatory System for Gait Monitoring Based on Wireless Sensorized Insoles. Sensors, 15(7):16589-16613. https://doi.org/10.3390/s150716589

[5] Cho, Soojin, and B. F. Spencer. (2015). Sensor Attitude Correction of Wireless Sensor Network for Acceleration - Based Monitoring of Civil Structures. Computer-Aided Civil and Infrastructure Engineering, 30(11):859-871. https://doi.org/10.1111/mice.12147

[6] Peng, Changhai, K. Qian, and C. Wang. (2015). Design and Application of a VOCMonitoring System Based on a ZigBee Wireless Sensor Network. IEEE Sensors Journal, 15(4): 2255-2268. https://doi.org/10.1109/JSEN.2014.2374156

[7] Yu, Yan, et al. (2016). A Study on Data Loss Compensation of WiFi-Based Wireless Sensor Networks for Structural Health Monitoring. IEEE Sensors Journal, 16(10): 3811-3818. https://doi.org/10.1109/JSEN.2015.2512846

[8] Yang, Z.L., Wu A. and Min. H.Q. (2016). Deployment of Wireless Sensor Networks for Oilfield Monitoring by Multiobjective Discrete Binary Particle Swarm Optimization. Journal of Sensors, 3:1-15. https://doi.org/10.1155/2016/9358358

[9] Zhang, C.J., et al. (2016). Tool Condition Monitoring and Remaining Useful Life Prognostic Based on a Wireless Sensor in Dry Milling Operations. Sensors, 16(6):795. https://doi.org/10.3390/s16060795

[10] Badiamelis, R, et al. (2015). Refrigerated fruit storage monitoring combining two different wireless sensing technologies: RFID and WSN. Sensors, 15(3): 4781-4795. https://doi.org/10.3390/s150304781

\section{Author}

Hongxia Sun is with Zhengzhou Shengda University of Economics, Business \& Management, Zhengzhou, China (sunhongxia421@163.com).

Article submitted 12 June 2017. Published as resubmitted by the author 18 July 2017. 